\title{
On URLLC Downlink Transmission Modes for MEC Task Offloading
}

\author{
Jinfei Wang, Yi Ma, Na Yi, and Rahim Tafazolli \\ Institute for Communication Systems (ICS), University of Surrey, Guildford, England, GU2 7XH \\ E-mail: (jinfei.wang, y.ma, n.yi,r.tafazolli)@surrey.ac.uk
}

\begin{abstract}
Multi-access edge computing for mobile computingtask offloading is driving the extreme utilization of available degrees of freedom (DoF) for ultra-reliable low-latency downlink communications. The fundamental aim of this work is to find latency-constrained transmission protocols that can achieve a very-low outage probability (e.g. $0.001 \%$ ). Our investigation is mainly based upon the Polyanskiy-Poor-Verdú formula on the finite-length coded channel capacity, which is extended from the quasi-static fading channel to the frequency selective channel. Moreover, the use of a suitable duplexing mode is also critical to the downlink reliability. Specifically, time-division duplexing (TDD) outperforms frequency-division duplexing (FDD) in terms of the frequency diversity-gain. On the other hand, FDD takes the advantage of having more temporal DoF in the downlink, which can be exchanged into the spatial diversity-gain through the use of space-time coding. Numerical study is carried out to compare the reliability between FDD and TDD under various latency constraints.
\end{abstract}

Index Terms-Multi-access edge computing (MEC), task offloading, downlink, aggregate encoding, ultra-reliable low-latency communications (URLLC).

\section{INTRODUCTION}

The use of multi-access edge computing (MEC) for mobile computing-task offloading has found many and still increasing applications such as connected automated vehicles [1], industrial automation [2], and virtual/augmented reality (VR/AR) [3]. The basic principle is to allow mobile computing devices (MCDs) to offload their computation-intensive tasks to the MEC through cellular radio uplink, with the aim of trading off the communication overhead for mobile computing-power saving as well as the overall computing-latency reduction. Current research activities in this domain mainly focus on the uplink procedure, which includes MEC selection [4], [5], uplink resource allocation [6], [7], as well as multiuser and multi-task management [8]-[10]. Their primary objective is to minimize the overall computing latency as well as MCD's energy consumption based upon the hypothesis of ultra-reliable and extremely low-latency downlink communication for the feedback of computing outcomes; for instance, most of published results were based on theoretically zerolatency and $100 \%$ reliability for the downlink when conducting their optimizations (for instance in [4], [6], [7], [9]). This hypothesis is driving the extreme physical-layer design for the downlink, which has to take into account various factors such as very limited time-domain degrees of freedoms (DoF), short messages, as well as random time of message arrival. While the uplink research is still going on, it is also the right time to work towards the hypothesis of ultra-reliable low-latency communications (URLLC) downlink.

The primary objective of URLLC downlink design is to find latency-constrained transmission protocols that can achieve a very low outage probability (e.g. $0.001 \%$ ). Earlier contributions in the URLLC domain have suggested the use of multiuser message aggregation and joint encoding [11]. It is anticipated that the message-aggregated encoding technique could bring significant improvements onto the reliability by leveraging the coding gain and channel frequency diversitygain, at the price of decoding complexity at MCDs, medium access control (MAC) complexities, as well as security and privacy concern [12]-[14]. Nevertheless, it remains unclear how the coding gain and diversity gain behave under various constraints of the temporal DoF; and how the lower MAC protocol should be designed to support the extreme use of available DoF in the time, frequency and spatial domains.

We strive to answer the above questions through numerical study. Our investigation is based on orthogonal frequency-division multiplexing (OFDM) systems, appreciating their wide applications and recognized advantages in wireless communications. Specifically, this work relies on the Polyanskiy-Poor-Verdú formula on the finite-length coded channel capacity-bound [15], which is extended, in this paper, from the quasi-static fading channel to space-time coded OFDM systems with the channel frequency-selectivity. The research focus is on the downlink protocol, with the uplink procedure being translated into the time or bandwidth uplink budget. The numerical results reveals that the messageaggregated encoding technique contributes considerable reliability enhancement to the downlink. However, our theoretic analysis also shows that due to the channel dispersion of frequency selective channels, the coding gain contributes few enhancement to the reliability. With respect to the uplinkdownlink duplexing mode, time-division duplexing (TDD) outperforms frequency-division duplexing (FDD) in terms of the channel frequency diversity-gain. On the other hand, FDD takes the advantage of having more temporal DoF in the downlink, which can be translated into the spatial diversitygain through the employment of space-time coding (STC). Numerical study is carried out to compare the reliability between FDD and TDD. 


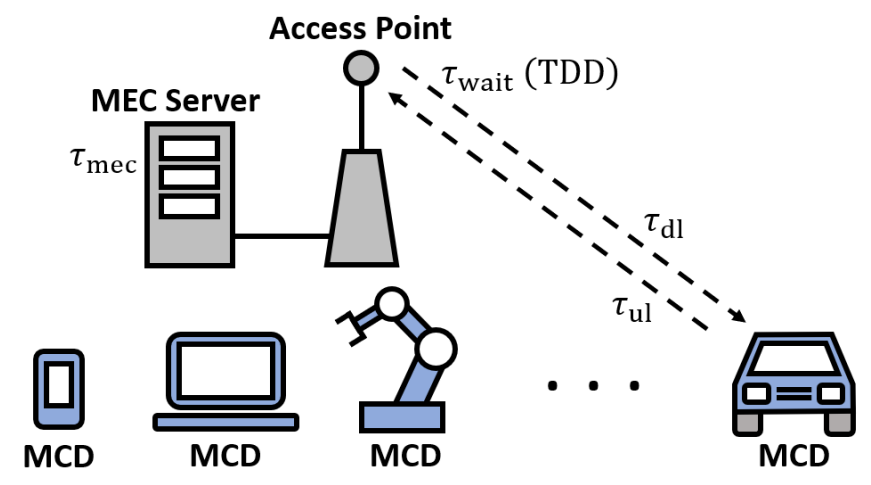

Fig. 1. System model and latency component of the MEC task offloading.

\section{System Model AND PRoblem Formulation}

Consider an access point (AP) connected with a set of MCDs, with each having single receive-antenna, through the OFDM air-interface with the total signal bandwidth $B_{\mathrm{s}}$. MCDs are uploading their computing tasks to the MEC through the AP. The time and bandwidth consumption of the uplink communication are $\tau_{\mathrm{ul}}$ and $B_{\mathrm{ul}}$, respectively. Denote $\tau_{\max }$ to be the overall latency constraint for each task offloading, and $\tau_{\text {mec }}$ to be the time consumption at the MEC. The AP has to complete the downlink procedure within the time constraint: $\tau_{\mathrm{dl}} \leq \tau_{\max }-\tau_{\mathrm{ul}}-\tau_{\mathrm{mec}}$. The bandwidth that can be utilized for the downlink is: $B_{\mathrm{dl}} \leq B_{\mathrm{s}}$. The setup of $\tau_{\mathrm{dl}}$ and $B_{\mathrm{dl}}$ is different when the MEC offloading procedure operates in different duplexing modes. Specifically, they are given by

$$
\begin{aligned}
\text { TDD: } \tau_{\mathrm{dl}} & =\tau_{\max }-\tau_{\mathrm{ul}}-\tau_{\mathrm{mec}}-\tau_{\mathrm{wait}}, \\
B_{\mathrm{dl}} & =B_{\mathrm{s}} \\
\text { FDD: } \tau_{\mathrm{dl}} & =\tau_{\max }-\tau_{\mathrm{ul}}-\tau_{\mathrm{mec}} \\
B_{\mathrm{dl}} & =B_{\mathrm{s}}-B_{\mathrm{ul}}
\end{aligned}
$$

where $\tau_{\text {wait }}$ denotes the delay in TDD, during which the downlink waits for the uplink to complete their transmissions (please see Fig. 1 and Fig. 2 for an illustrative explanation). Note that we use common notations $\tau_{\mathrm{dl}}$ and $B_{\mathrm{dl}}$ for TDD and FDD mainly for the notation simplicity; and later on they will serve as variables in the Polyanskiy-Poor-Verdú formula.

Computing outcomes of the MEC are represented in form of short messages, say $K$ bit/message. For every downlink transmission, the MEC will generate $N(>0)$ short messages, where $N$ is a random integer with its probability distribution determined by the MEC capacity and upper layer protocols. When the AP encodes $N$ messages together and send them to all MCDs in one go, the downlink data-rate is given by

$$
R=\frac{(N)(K)}{\left(\tau_{\mathrm{dl}}\right)(B)} .
$$

where $B$ is the signal bandwidth allocated for the $N$ messages ( $\left.B \leq B_{\mathrm{dl}}\right)$. Defining $\bar{R}\left(\tau_{\mathrm{dl}}, B, N\right)$ to be the instantaneous

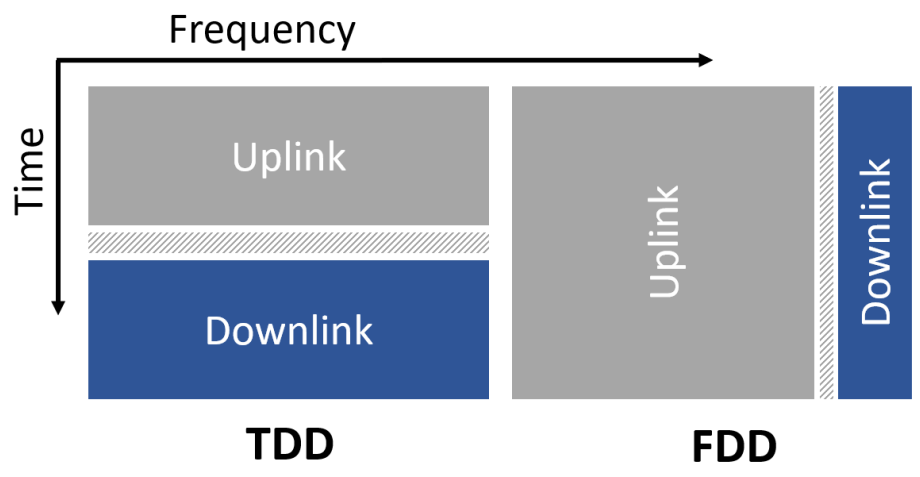

Fig. 2. The comparison of TDD and FDD for a MEC system.

maximum achievable-rate for the downlink, the outage probability is measured by

$$
p_{\text {out }}=\operatorname{Prob}\left(\bar{R}\left(\tau_{\mathrm{dl}}, B, N\right)<R\right) .
$$

Here, each AP-to-MCD channel is assumed to be i.i.d., and thus they have the identical outage probability in fading channels. Moreover, it is perhaps worth noting that $p_{\text {out }}$ is the outage probability of every single AP-to-MCD link. In the case of extreme URLLC, the AP normally has no temporal DoF to offer a retransmission [16], and thus a single-link outage probability is more meaningful than a system outage probability.

Given the above system description, the research problems highlighted in Sec. I are equivalent to: Q1) investigating the behavior of coding and diversity gains in terms of downlink outage probability; and Q2) comparing the performance between FDD and TDD under various temporal DoF constraints.

\section{Fundamental PRinciples FOR the DOWNLINK RELIABILITY AND LATENCY}

\section{A. Discrete-time Equivalent Representation of DoF in OFDM}

Prior to study the channel capacity, we shall translate those time and bandwidth notations in Sec. II from the continuoustime representations into their discrete-time counterparts.

Consider a generic case where the AP sends an aggregated message $\mathbf{s}$, with the length $(N)(K)$ bits. Denote $\Delta B$ to be the subcarrier spacing in the OFDM system. There are totally $M=\lfloor(B) /(\Delta B)\rfloor$ subcarriers in the system $(\lfloor\cdot\rfloor$ denotes the integer floor). Given $T_{\mathrm{cp}}$ the time duration of the cyclic prefix $(\mathrm{CP})$, the downlink can have a maximum of $J=\left\lfloor\left(\tau_{\mathrm{dl}}\right) /\left(T_{\mathrm{cp}}+\Delta B^{-1}\right)\right\rfloor$ OFDM symbols. Correspondingly, the downlink data-rate becomes

$$
R=\frac{(N)(K)}{\left(\tau_{\mathrm{dl}}\right)(M)} .
$$

And the discrete-time equivalent form of the frequencydomain received signal in the downlink is represented by

$$
\mathbf{y}_{j}=\mathcal{D} \mathbf{x}_{j}+\mathbf{v}_{j}, \quad j=0, \ldots, J-1
$$

where $\mathbf{y}_{j}$ stands for the received OFDM signal vector with the size of $(M) \times(1), \mathbf{x}_{j}$ for the $(M) \times(1)$ transmitted signal 
vector which is the encoded version of $\mathbf{s}, \mathcal{D}$ for the diagonal channel matrix with the channel frequency response (CFR), denoted by $\left[H_{0}, \ldots ., H_{M-1}\right]^{T}$, in its diagonal $\left([\cdot]^{T}\right.$ stands for the matrix/vector transpose), and $\mathbf{v}_{j}$ for the additive white Gaussian noise (AWGN).

Let's assume that the channel matrix $\mathcal{D}$ does not vary considerably within $J$ OFDM symbols. Given the knowledge of the channel matrix $\mathcal{D}$, each MCD can perform decoding individually based upon $\mathbf{y}_{j}$, i.e.,

$$
\hat{\mathbf{s}}=\operatorname{decode}\left(\mathbf{y}_{j} \mid \mathcal{D}\right), \forall j
$$

where $\hat{\mathbf{s}}$ is the decoded version of $\mathbf{s}$. When the decoding is successful (i.e. no decoding error found), each MCD picks up their desired information bits from $\hat{\mathbf{s}}$. It is worthwhile to note that each MCD normally has a different observation of $\mathbf{y}$ and $\mathcal{D}$ due to channel fading behavior as well as their local noise. Since each MCD performs decoding individually, our mathematical analysis will be mainly focused on the singlelink level. The outage probability (2) can now be represented by

$$
p_{\text {out }}=\operatorname{Prob}(\bar{R}(J, M, N)<R) .
$$

\section{B. Polyanskiy-Poor-Verdú Formula for Aggregated Encoding}

We start from the AWGN scenario for the Polyanskiy-PoorVerdú formula.

Theorem 1 (a represented version from the formula in [15]). Suppose $\mathcal{D}$ to be an identity channel matrix. Given a decoding error probability $\epsilon$, the maximum achievable-rate $\bar{R}$ for the channel model (4) is approximately

$$
\bar{R} \approx C(\gamma)-\sqrt{\frac{V(\gamma)}{L}} \frac{\mathcal{Q}^{-1}(\epsilon)}{\ln (2)}
$$

where $\gamma$ stands for the instantaneous signal-to-noise ratio (SNR), $C(\gamma)=\log _{2}(1+\gamma), V(\gamma)=1-(1+\gamma)^{-2}, \mathcal{Q}(\cdot)$ for the Gaussian- $Q$ function, and $L$ for the number of symbols going through the channel; $L=(J)(M)$ in (4).

When $\mathcal{D}$ represents the frequency-selective channel, the channel capacity $C(\gamma)$ becomes

$$
C(\gamma)=\frac{1}{M} \log _{2}\left(\operatorname{det}\left(\mathbf{I}+\gamma \mathcal{D} \boldsymbol{\Xi} \mathcal{D}^{\mathrm{H}}\right)\right),
$$

where $\boldsymbol{\Xi}=\mathbb{E}\left(\mathbf{x}_{j} \mathbf{x}_{j}^{H}\right)$ is the signal covariance matrix, $\mathbb{E}(\cdot)$ the expectation, and $(\cdot)^{H}$ the matrix/vector Hermitian. It is worthwhile to emphasize that $\boldsymbol{\Xi}$ is not a diagonal matrix due to the element-wise correlation introduced by the channel encoding.

Define a Hermitian matrix $\mathbf{A}=\mathcal{D} \boldsymbol{\Xi} \mathcal{D}^{\mathrm{H}}$. We can apply the eigenvalue decomposition (EVD) on $\mathbf{A}: \mathbf{U}^{H} \mathbf{A} \mathbf{U}=\boldsymbol{\Lambda}$, where $\mathbf{U}$ is the unitary matrix, and $\boldsymbol{\Lambda}$ the diagonal matrix with all eigenvalues in its diagonal. Denoting $\lambda_{i}, \quad i=0, \ldots, \bar{M}-1$, to be eigenvalues of $\mathbf{A}(\bar{M} \leq M)$, (8) is equivalent to

$$
C(\gamma)=\frac{1}{M} \sum_{i=0}^{\bar{M}-1} \log _{2}\left(1+\gamma \lambda_{i}\right) .
$$

According to $[17,(97)]$, the channel dispersion $V(\gamma)$ is given by

$$
V(\gamma)=\bar{M}-\sum_{i=0}^{\bar{M}-1}\left(1+\gamma \lambda_{i}\right)^{-2}
$$

Plugging (9)-(10) into (7) yields the approximation of the maximum achievable rate $\bar{R}(\gamma)$ for the frequency-selective channel with finite block-length.

\section{Performance Evaluation for Message Aggregated Encoding and Duplexing}

1) Gain of the multiuser message-aggregated encoding: Consider a baseline system where $N$ MCDs are receiving computing outcomes from the AP in the frequency division multiple-access (FDMA) manner. Each MCD occupies $M_{\mathrm{s}}$ consecutive subcarriers. Assuming the channel to be approximately frequency non-selective for each MCD, we can use (7) to immediately have

$$
\bar{R}_{n} \approx \log _{2}\left(1+\gamma_{n}\right)-\sqrt{\frac{V_{\mathrm{s}}\left(\gamma_{n}\right)}{(J)\left(M_{\mathrm{s}}\right)}} \frac{\mathcal{Q}^{-1}(\epsilon)}{\ln (2)}
$$

where $\gamma_{n}$ is the instantaneous-SNR within the $n^{\text {th }}$ MCD's operating band, and $V_{\mathrm{s}}\left(\gamma_{n}\right)=M_{\mathrm{s}}\left(1-\left(1+\gamma_{n}\right)^{-2}\right)$ is the channel dispersion for the quasi-static OFDM channel. Plugging $V_{\mathrm{s}}\left(\gamma_{n}\right)$ into (11) leads to the identical result with (7), where the term $M_{\mathrm{s}}$ is canceled out. The exactly same result can be also derived when the multiuser message-aggregated encoding is applied onto $N$ MCDs. Thus we can draw the following conclusion.

Corollary 1. In the flat OFDM channel, the maximum achievable-rate $\bar{R}$ increases only with the number of OFDM symbols (i.e. the latency J); and it is not relevant to the number of subcarriers (i.e. $M$ ) involved in the communication.

Therefore, in the flat OFDM channel, there is a tradeoff between the outage probability (6) and the number of OFDM symbols $J$ (downlink latency); and the parameter pair $(M, N)$ has no impact on the outage probability. Corollary 1 means multiuser message-aggregated encoding cannot bring extra coding gain. Nevertheless, it can be employed to reduce the decoding error probability $\epsilon$. This is important because the decoding error probability $\epsilon$ should be reduced to a certain level such that it would not become the bottleneck of the outage probability.

2) Gain of the channel frequency diversity: When multiuser messages are aggregated and jointly encoded, $\mathcal{D}$ is practically not an identity matrix; and then, the result (9)-(10) should apply to (7). Therein, (9) is the standard formula for the OFDM channel capacity, which shows the fundamental multiplexingdiversity tradeoff in the frequency domain. To facilitate our discussion, we assume:

A1) The CFR at different MCD's band is approximately independent fading ;

A2) There exists an encoding scheme that achieves the maximum frequency diversity-gain. 
The capacity formula (9) can be simplified into

$$
C(\gamma)=\frac{\left(M_{\mathrm{s}}-1\right)}{M_{\mathrm{s}}} \log _{2}\left(1+\frac{1}{N} \sum_{n}^{N-1} \gamma_{n}\right)
$$

where $\bar{M}=\left(M_{\mathrm{s}}-1\right)(N)$ is employed in the simplification procedure (derivation omitted due to the space limit). Our further concern is on the rate-loss term in (7), with particular interest in the factor

$$
\begin{aligned}
\sqrt{\frac{V(\gamma)}{L}} & =\sqrt{\frac{\bar{M}-\sum_{i=0}^{\bar{M}-1}\left(1+\gamma \lambda_{i}\right)^{-2}}{(J)\left(M_{\mathrm{s}}\right)(N)}} \\
& =\sqrt{\frac{\left(M_{\mathrm{s}}-1\right)\left(1-\left(1+\frac{1}{N} \sum_{n}^{N-1} \gamma_{n}\right)^{-2}\right)}{(J)\left(M_{\mathrm{s}}\right)}}
\end{aligned}
$$

For the practical case where $M_{\mathrm{s}} \approx\left(M_{\mathrm{s}}-1\right)$, we can have the following maximum achievable-rate

$$
\bar{R} \approx \log _{2}(1+\bar{\gamma})-\sqrt{\frac{\left(1-(1+\bar{\gamma})^{-2}\right)}{J}} \frac{\mathcal{Q}^{-1}(\epsilon)}{\ln (2)}
$$

where $\bar{\gamma}=\frac{1}{N} \sum_{n}^{N-1} \gamma_{n}$. Then, it is a bit tedious but trivial to justify that the diversity-combined SNR $\bar{\gamma}$ contributes positively to the outage probability, with the channel frequency diversity-order of $N$. This diversity order shall reduce when CFRs at different MCD's bands are correlated.

3) Exchanging temporal DoF into the spatial diversity: The STC technique can be employed to exchange the temporal DoF into the spatial diversity-gain. For instance, the Alamouti's scheme [18] can introduce the transmitter spatial diversitygain in order of 2 by halving the temporal-DoF (i.e. $J$ ). More generally, linear dispersion code (LDC) can further improve the spatial diversity gain at higher cost of the temporal-DoF; for instance, $\mathrm{LDC}$ can increase the transmitter spatial diversityorder to 4 by reducing the temporal-DoF to $(J) /(8)$ [19]. Denote $\alpha$ to be the transmitter spatial diversity order and $\beta^{-1}$ the loss of the temporal-DoF $(\beta \geq \alpha)$. The maximum achievable-rate is given by

$$
\bar{R} \approx \log _{2}\left(1+\bar{\gamma}_{\mathrm{stc}}\right)-\sqrt{\frac{\beta\left(1-\left(1+\bar{\gamma}_{\mathrm{stc}}\right)^{-2}\right)}{J}} \frac{\mathcal{Q}^{-1}(\epsilon)}{\ln (2)}
$$

where $\bar{\gamma}_{\mathrm{stc}}=\alpha^{-1} \sum_{t=0}^{\alpha-1} \bar{\gamma}_{t}$, and $\bar{\gamma}_{t}$ denotes the effective combined-SNR in the $t^{\text {th }}$ spatial channel. It can be observed that STC results in the rate-loss in factor of $\sqrt{\beta}$, which could be really considerable for LDC (e.g. $\beta=8$ ). Adopting STC schemes where $\beta$ is very high may lead to the degradation of reliability.

\section{NUMERICAL RESULTS FOR OFDM URLLC WITH TDD OR FDD}

Numerical evaluations are carried out to evaluate the performance of both FDD and TDD. Due to the strict latency requirement, $\Delta B$ is considered to be $30 \mathrm{KHz}$ [20]. Moreover, $T_{\mathrm{cp}}$ is considered as one eighth of the OFDM symbol duration. $B_{\mathrm{s}}$ is considered as $99.84 \mathrm{MHz}$, i.e. 3328 subcarriers. For TDD, each MCD is allocated 104 subcarriers. For FDD, the

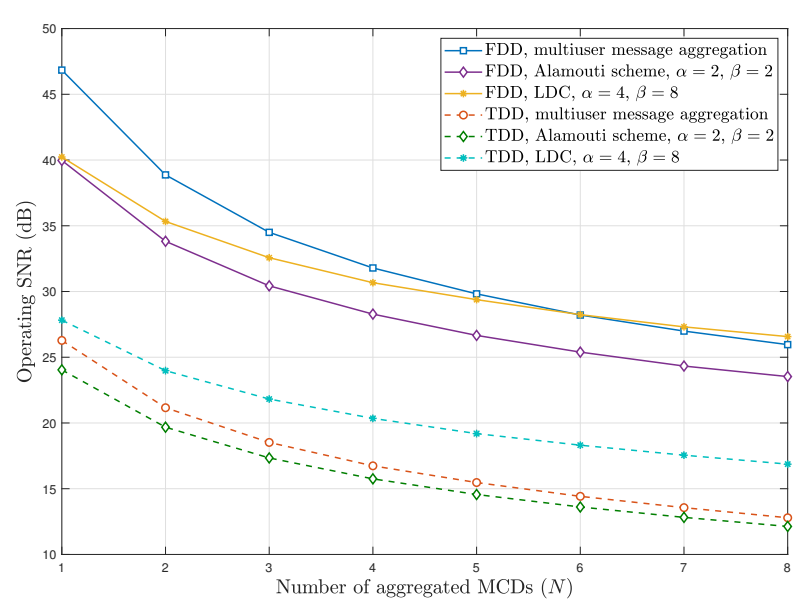

Fig. 3. The operating SNR (the SNR to achieve $10^{-5}$ outage probability) of FDD and TDD when adopting multiuser message aggregation and STC.

downlink bandwidth $B_{\mathrm{dl}}$ is $15.36 \mathrm{MHz}$, i.e. 512 subcarriers, while each MCD is allocated 16 subcarriers. For FDD, $\tau_{\mathrm{dl}}=1$ $\mathrm{ms}$, while for TDD, $\tau_{\mathrm{dl}}+\tau_{\text {wait }}=1 \mathrm{~ms}$. Consider a pessimistic case in TDD, where the downlink needs to wait for one uplink transmission time interval (TTI) to start the transmission. The uplink TTI is considered to be 7 OFDM symbols as in the long-term evolution (LTE) system, so $\tau_{\text {wait }} \approx 0.25 \mathrm{~ms}$. The length of one downlink message $K$ is 32 bytes, i.e. 256 bits, as in the URLLC requirement [21]. The evaluations are structured into two experiments. Due to the space limit, their results are contained together in Fig. 3.

Experiment 1: The objective of this experiment is to investigate the reliability improvement of adopting multiuser aggregation. Fig. 3 shows the operating SNR (the SNR to achieve $10^{-5}$ outage probability) of both FDD and TDD. It is shown that multiuser message aggregation can bring significant reliability improvement. When $N=8$, the operating SNR of FDD and TDD has a reduction of $20.9 \mathrm{~dB}$ and $13.5 \mathrm{~dB}$ respectively. It is also shown that as $N$ increases, the decrement of operating SNR decreases gradually. This is because the frequency diversity order $\bar{M}$ is determined by the channel tap delay and is much smaller than the number of subcarriers $M$. As $M$ increases linearly to $N$, the frequency diversity gradually approaches its limit, and the reliability improvement increment per MCD decreases gradually. In this case, TDD's frequency diversity order is closer to the limit, and its frequency diversity gain should be less significant than FDD. This phenomenon can also be observed in Fig. 3 .

Moreover, TDD outperforms FDD in terms of reliability. Although their performance gap keeps decreasing as $N$ increases, TDD's operating SNR is still $13 \mathrm{~dB}$ lower than FDD when $N=8$. This is because TDD has significantly more frequency diversities than FDD. When $N=6$, the operating SNR of TDD is already lower than $15 \mathrm{~dB}$. While for FDD, the operating SNR is $26 \mathrm{~dB}$ when $N=8$. 
Experiment 2: The objective of this experiment is to investigate the behavior of spatial diversity gain when adopting STC. Two STC schemes are considered here: Alamouti scheme where the transmitter diversity $\alpha=2$ and the rate-loss factor $\beta=2$ due to temporal DoF payment, as well as the LDC scheme where $\alpha=4$ and $\beta=8$. It is shown in Fig. 3 that when adopting Alamouti scheme, the operating SNR is improved for around $4 \mathrm{~dB}$ and $1 \mathrm{~dB}$ for FDD and TDD respectively. These improvements are due to the extra frequency diversity order introduced by the spatial diversity. Moreover, the reliability improvement for TDD is smaller than FDD. This is because TDD has less temporal DoF, which leads to more rate-loss in (16). Such result also reveals that adopting Alamouti scheme cannot make significant reliability improvement for TDD.

On the other hand, the performance of adopting LDC is showing obvious degradation compared to adopting Alamouti scheme. This is because the high temporal DoF payment $(\beta=8)$ leads to severe rate-loss. For TDD, adopting LDC even leads to $4 \mathrm{~dB}$ performance degradation compared to not adopting STC. Such result shows that high temporal DoF payment cannot improve the reliability for URLLC transmissions. Moreover, When adopting STC, TDD still outperforms FDD in terms of reliability.

\section{CONCLUSiOnS}

This paper has presented the fundamental behavior of coding gain and diversity gains under URLLC performance requirements as well as comparison between duplexing modes for MEC downlink transmissions. For this purpose, the Polyanskiy-Poor-Verdú formula on the finite-blocklength coded channel capacity bound has been extended from quasistatic fading channel to the frequency selective channel. Through numerical analysis, it was found that multiuser message aggregation can significantly improve the reliability. However, theoretical analysis reveals that these improvements are contributed by frequency diversity gain alone, without coding gain. When exchanging the temporal DoF for spatial diversity through adopting STC, the reliability improvement depends on the temporal DoF payment. For low temporal DoF payment, the downlink reliability can be improved. But for high temporal DoF payment, adopting STC could lead to negative effect on the reliability. Moreover, in all numerical examples, TDD significantly outperforms FDD (around $10 \mathrm{~dB}$ or above), taking advantage of extra frequency diversity.

\section{ACKNOWLEDGMENT}

This work is funded in part by the UK 5G Innovation Centre (5GIC), and in part by EU H2020 5G-DRIVE programme.

\section{REFERENCES}

[1] B. Gu and Z. Zhou, "Task offloading in vehicular mobile edge computing: A matching-theoretic framework," IEEE Vehicular Technology Magazine, vol. 14, no. 3, pp. 100-106, Sep. 2019.
[2] I. A. Tsokalo, H. Wu, G. T. Nguyen, H. Salah, and F. H. P. Fitzek, "Mobile edge cloud for robot control services in industry automation," in 2019 16th IEEE Annual Consumer Communications Networking Conference (CCNC), Jan. 2019, pp. 1-2

[3] X. Yang, Z. Chen, K. Li, Y. Sun, and H. Zheng, "Optimal task scheduling in communication-constrained mobile edge computing systems for wireless virtual reality," in 2017 23rd Asia-Pacific Conference on Communications (APCC), Dec. 2017, pp. 1-6.

[4] H. Guo, J. Liu, and J. Zhang, "Efficient computation offloading for multiaccess edge computing in 5G HetNets," in 2018 IEEE International Conference on Communications (ICC), May 2018, pp. 1-6.

[5] P. Zhang, J. Yang, and R. Fan, "Energy-efficient mobile edge computation offloading with multiple base stations," in 15th International Wireless Communications Mobile Computing Conference (IWCMC), June 2019, pp. 255-259.

[6] S. Han, X. Xu, S. Fang, Y. Sun, Y. Cao, X. Tao, and P. Zhang, "Energy efficient secure computation offloading in NOMA-based mMTC networks for IoT," IEEE Internet of Things Journal, vol. 6, no. 3, pp. 5674-5690, June 2019.

[7] X. Yang, X. Yu, H. Huang, and H. Zhu, "Energy efficiency based joint computation offloading and resource allocation in multi-access MEC systems," IEEE Access, vol. 7, pp. 117 054-117062, Sep. 2019.

[8] S. Wang, C. Pan, and C. Yin, "Joint heterogeneous tasks offloading and resource allocation in mobile edge computing systems," in 2018 10th International Conference on Wireless Communications and Signal Processing (WCSP), Oct. 2018, pp. 1-6.

[9] B. Yang, X. Cao, J. Bassey, X. Li, T. Kroecker, and L. Qian, "Computation offloading in multi-access edge computing networks: A multitask learning approach," in 2019 IEEE International Conference on Communications (ICC), May 2019, pp. 1-6.

[10] J. Zhang, M. Wu, and M. Zhao, "Joint computation offloading and resource allocation in C-RAN with MEC based on spectrum efficiency,' IEEE Access, vol. 7, pp. 79 056-79068, June 2019.

[11] L. Hoang, E. Uhlemann, and M. Jonsson, "Relaying with packet aggregation for half-duplex all-to-all broadcast in time-critical wireless networks," in 2017 IEEE Globecom Workshops (GC Wkshps), Dec. 2017, pp. 1-6.

[12] Z. Guo and X. Ding, "Low energy-consuming cluster-based algorithm to enforce integrity and preserve privacy in data aggregation," in 2014 13th International Symposium on Distributed Computing and Applications to Business, Engineering and Science, Nov. 2014, pp. 152-156.

[13] S. Jian, D. Liu, X. Chen, J. Li, N. Kumar, and P. Vijayakumar, "Secure real-time traffic data aggregation with batch verification for vehicular cloud in VANETs," IEEE Transactions on Vehicular Technology, pp. 1-1, Oct. 2019.

[14] H. S. Annapurna and M. Siddappa, "Secure data aggregation with fault tolerance for wireless sensor networks," in 2015 International Conference on Emerging Research in Electronics, Computer Science and Technology (ICERECT), Dec. 2015, pp. 29-33.

[15] Y. Polyanskiy, H. V. Poor, and S. Verdú, "Channel coding rate in the finite blocklength regime," IEEE Trans. Inf. Theory, vol. 56, no. 5, pp. 2307-2359, May 2010.

[16] P. Marsch, I. Silva, Ö. Bulakci, M. Tesanovic, S. Ayoubi, and M. Säily, "Emerging network architecture and functional design considerations for 5G radio access," Trans. Emerg. Telecommun. Technol., vol. 27, pp. 1168-1177, Sep. 2016.

[17] W. Yang, G. Durisi, T. Koch, and Y. Polyanskiy, "Quasi-static multiple antenna fading channels at finite blocklength," IEEE Trans. Inf. Theory, vol. 60, no. 7, pp. 4232-4265, Jul. 2014

[18] S. M. Alamouti, "A simple transmit diversity technique for wireless communications," IEEE Journal on Selected Areas in Communications, vol. 16, no. 8, pp. 1451-1458, Oct. 1998.

[19] B. Hassibi and B. M. Hochwald, "High-rate codes that are linear in space and time," IEEE Transactions on Information Theory, vol. 48, no. 7, pp. 1804-1824, July 2002.

[20] Physical channels and modulation (Release 15) - 3GPP TS 38.211 V15.7.0, 3rd Generation Partnership Project Std.

[21] Service requirements for next generation new services and markets (Release 15) - 3GPP TS 22.261 V17.1.0, 3rd Generation Partnership Project Std. 